\title{
The role of the visual arts in the resilience of people living with dementia in care homes
}

\author{
Andrew Newman ${ }^{1 \star}$, Anna Goulding ${ }^{2}$, Bruce Davenport ${ }^{1}$ and Gill Windle ${ }^{3}$ \\ ${ }^{1}$ School of Arts and Cultures, Newcastle University, Newcastle upon Tyne, UK, ${ }^{2}$ Institute of Health and Society, \\ Sir James Spence Institute, Newcastle University, Royal Victoria Infirmary, Newcastle upon Tyne, UK and \\ ${ }^{3}$ Dementia Services Development Centre, Institute of Medical and Social Care Research, Bangor University, UK \\ ${ }^{*}$ Corresponding author. Email: andrew.newman@ncl.ac.uk
}

(Accepted 20 April 2018; first published online 27 June 2018)

\begin{abstract}
This study responds to a gap in the literature relating to the resilience of people living with dementia in care homes. We applied an ecopsychosocial framework of resilience, theorising that sources of resilience may be personal, social and structural. Visual arts enrichment activities were examined to see how they might provide opportunities for resilience. The data used for this study were qualitative and originated from people with dementia aged between 70 and 99 years old $(\mathrm{N}=48)$ living in four care homes in North East England, United Kingdom and staff/carers/family members $(\mathrm{N}=37)$. The results showed that visual arts enrichment activities supported the resilience of those with dementia through creative expression, increased communication, improved selfesteem, and influenced relationships with carers and family members. It is concluded that even those with advanced dementia are capable of demonstrating resilience which can be supported by, and explored through, visual arts enrichment activities.
\end{abstract}

Keywords: arts enrichment activities; dementia; care homes; resilience; older people; carers

\section{Introduction}

At present there is little knowledge about how resilience in people in later life with dementia might be understood or even whether such an outcome is possible. An understanding of this is important to improving the care of the $850,000^{1}$ people living with dementia in the United Kingdom (UK), which is one of the aims of the government's UK Dementia Challenge. This paper addresses the topic through the analysis of qualitative data collected from four care homes in North East England, UK, originating from the Dementia and Imagination ${ }^{2}$ research project. This examined how visual arts enrichment activities might improve the quality of life for people in later life with dementia and their carers. This is a UK national project funded by the UK Economic and Social Research Council and the UK Arts and Humanities Research Council (reference AH/J011029/1) through the

(c) Cambridge University Press 2018. This is an Open Access article, distributed under the terms of the Creative Commons Attribution licence (http://creativecommons.org/licenses/by/4.0/), which permits unrestricted re-use, distribution, and reproduction in any medium, provided the original work is properly cited. 
Connected Communities Programme. ${ }^{3}$ The definition of resilience adopted for this study is 'the process of effectively negotiating, adapting to, or managing significant sources of stress or trauma' (Windle 2011: 152).

The reason for the focus upon care homes is that they represent a context that involves a series of challenges to the resilience of residents. This is illustrated by the qualitative study of the experience of living with dementia in residential care by Claire et al. (2008). Participants demonstrated feelings of isolation and alienation and complained that they 'felt confined and wanted more independence' (2008: 717) and were unable to 'exert any control over their situation' (2008: 714). There are also threats to selfhood ${ }^{4}$ associated with limited opportunities for selfexpression and social interaction. In Buettner and Fitzsimmons' (2003: 215) study of long-term care residents with dementia, 45 per cent $(\mathrm{N}=107)$ of the sample had 'little or no facility activity', meaning that there was little in the way of enrichment activities being undertaken. These aspects of life in a care home might be expected to negatively influence the resilience of people with dementia living in this environment. This makes care homes a unique environment to study resilience in those with more advanced dementia.

This paper is organised in the following way. Firstly, the theoretical framework adopted for the analysis is presented followed by an account of the methodology, data and visual arts enrichment activity. This is followed by a description of the analysis, the results of which are organised around a series of themes identified from the qualitative data, a discussion and conclusion.

\section{Theoretical framework}

\section{Conceptualising resilience}

Many disciplines use the term resilience, but do not always apply it in the same way. This creates challenges for measurement and differences in understanding. After undertaking a review and concept analysis of 271 research papers, Windle defined resilience as:

The process of effectively negotiating, adapting to, or managing significant sources of stress or trauma. Assets and resources within the individual, their life and environment facilitate this capacity for adaptation and 'bouncing back' in the face of adversity. Across the life course, the experience of resilience will vary. (Windle 2011: 152)

This definition, which is adopted by this paper, is inspired by the ecological theory of human development (Bronfenbrenner 1994), which has since been developed into an ecological framework of resilience. The framework has been used to conceptualise resilience across the lifespan (Windle 2012), to explore familial care-giving (Donnellan, Bennett and Soulsby 2015; Windle and Bennett 2011) and to explore the lives of older Columbians living in poverty (Bennett et al. 2016). It acknowledges that the resources for a good outcome, despite adversity, are operationalised across micro/individual, meso/community and macro/wider society levels.

In a similar approach to that given above, Wild, Wiles and Allen (2013) identified a distinction between individual resilience, which may be the result of a lifetime 
of accumulating skills and knowledge, as represented by their human capital and that which originates outside the individual from the family, household, neighbourhood, community and wider society, as represented by their social capital. Wild, Wiles and Allen (2013) also noted that identifying exactly what constitutes risk is problematic because what may constitute risk for one individual may not for another. Those recruited to this study exhibited risks experienced by people in later life such as 'dementia, depression and physical frailty' (Wild, Wiles and Allen 2013: 138). They also stated that there is as of yet no agreement as to what might be 'distinctive and unique' (2013: 141) about the resilience of people in later life rather than just being associated with it. ${ }^{5}$ An important conclusion from Masten's (2001: 227) work on resilience is that it is an ordinary occurrence and that 'it usually arises from the normative functions of human adaptational systems'. He argued that the greatest threats to human development are those that compromise these protective systems.

In order to demonstrate resilience, in this particular research context having dementia to varying degrees was the adversity to overcome. Additionally, the person involved had to be seen to be 'doing all right' (Braudy Harris 2008: 45) despite the challenges they faced. Clearly, a judgement over whether a respondent is 'doing all right' or not is a subjective one and is often made by carers and family members. Assessments of this might vary over time both in terms of possible longer-term cognitive decline and the short-term changes in symptoms, which can occur hour-by-hour, often experienced by those with dementia. The literature has little to say about the process of adaptation itself, which is described as 'ordinary magic' by Masten (2001) because of its everyday nature. For example, Wild, Wiles and Allen (2013) discussed external resources, such as those available through social networks, but not how those resources are understood and operationalised as social contacts do not guarantee access to resources that might be used for resilience purposes.

Studies of the resilience of older people with dementia are few in number (e.g. Braudy Harris 2008; Claire et al. 2011; Clarke and Bailey 2016), and this work tends to focus on those in the early stages of the condition. ${ }^{6}$ Most of the respondents represented in these studies are capable of answering questions from interview schedules and they are able to reflect on their situation. Involving participants who have more advanced dementia in research on resilience, such as those who have become non-verbal and/or are experiencing severe cognitive decline, means that traditional interview schedules cannot be relied upon. Therefore, other methods, such as interviews with carers or a more ethnographic approach such as observation and the use of videos of activities, can be seen as providing more holistic representational data.

The concept of resilience is attractive to gerontology as it provides an alternative to the paradigm of successful ageing (Rowe and Kahn 1987). Successful ageing is seen to consist of 'avoiding disease and disability', 'engagement with life' and having 'high cognitive and physical function', which means that many people in later life would be excluded because of their life circumstances. Resilience is potentially achievable by all people regardless of their situation, including those with dementia. Authors such as Braudy Harris (2008) suggested that it should be the objective of all as they age. 


\section{The contribution of the arts to the resilience of people in later life}

There are a small number of studies that link participation in the arts with resilience in people in later life. For example, McFadden and Basting (2010) and Roush et al. (2011) suggested that the main ways that arts activities may contribute to resilience is to 'encourage individual expression and to strengthen social connections' (McFadden and Basting 2010: 151) and to 'increase a sense of mastery and control' (2010: 158). There is also discussion of the role of creative activity (whether in an arts activity or not), which might be associated with resilience and is understood as a process whereby the individual seeks an original solution to a problem or challenge at hand (Mariske and Willis 1998). Fisher and Specht (1999: 459) stated that 'older people who have developed their creative abilities in some domains can apply these skills to other domains and are more able to cope with the many challenges and problems that occur in later life'. However, much of the above work is theoretical rather than empirical, which is a gap in the literature that this paper addresses.

There is a growing body of literature that links arts engagement with the wellbeing of people in later life (e.g. Cohen 2006), which can be linked to work on resilience. Evidence for this comes from the New Dynamics of Ageing programme of research ${ }^{7}$ which suggested that the arts can facilitate social connectivity, help address negative societal meta-narratives (Murray et al. 2014), and construct socially situated identities that respond to individual and community challenges (Newman, Goulding and Whitehead 2013), providing resources for resilience. Similarly, Young et al. (2015) have undertaken a systematic review of the literature on the impact of community-based arts and health interventions on cognition in those with dementia and conclude that such activities have positive effects. However, they also recognised the limitations of relying on positivist methodologies when dealing with art or creative approaches. It is suggested by Basting and Killick (2003) that creative activities are particularly important for people in later life with dementia, as they may have lost many normal opportunities for self-expression.

\section{Methodology, data and visual arts enrichment activity}

The research protocol for the Dementia and Imagination project has been published by Windle et al. (2016) and the process of deciding upon various methodological approaches by Newman et al. (2016). These provide details of the research project and the wide range of academic and community partners that were involved in the work. The research took place between August 2013 and August 2017 with data collection being completed by January 2016 .

\section{Study design}

The approach consisted of mixed methods, primarily qualitative and quantitative. It also viewed creative practice as a way of providing new perspectives on a topic and it is seen as a methodology in itself (Hope 2016). The analysis of a range of visual arts enrichment activities run by participatory artists, described below, enabled the processes of adaptation leading to resilience within the social-cultural context of the care home (Downs 2000) to be analysed. This was in an attempt to improve the lives of people in later life with dementia rather than preventing or treating the condition. 


\section{Setting and participants}

The arts enrichment activities took place in four different care homes in North East England, UK, and participants were included according to the following criteria:

- Diagnosis of dementia or evidence of age-related memory impairment.

- A resident in the chosen care home in North East England, UK.

Potential participants were excluded if they had a recent or current episode of major mental illness, were at the end of life or terminally ill, had a debilitating illness that would preclude regular attendance, had a severe uncorrected sensory or communication difficulty, or were completely unable to communicate verbally.

Only three of the care home participants had the capacity to consent to take part and their involvement reflected their interest either in being involved in a social activity or/and an interest in art. However, at recruitment some thought the activity would involve learning to draw or paint and this was off-putting because of an assumed lack of skill on the behalf of the participant. Providing a description of the activities dispelled this (see below).

\section{Procedure}

The study received UK National Health Service ethical approval from the North Wales Research Ethics Committee - West (reference number 13/WA/0365). The research and the visual arts enrichment activities were undertaken within a broad ethical approach (Wiles 2013). It was recognised that the arts enrichment activities and research methods, described below, were not risk free. A recruitment protocol was developed which emphasised that participants could pull out of the project at any time without explanation. The provisions of the UK Mental Capacity Act 2005 were followed and if the potential participant was unable to make an informed choice, an authorised family member or carer provided consent on their behalf, as is noted in the research protocol (Windle et al. 2016). Research staff and artists undertook training by the lead investigators and this covered the Mental Capacity Act 2005, informed consent, dementia awareness and the study protocol procedures, including data collection methods and data management. All the artists and those responsible for data collection had experience of working with older people, although not all had worked with older people with dementia.

Newman and Goulding were responsible for data collection. Both had experience in undertaking similar projects and had worked with people in later life with dementia. A relationship was established with the care homes before the study commenced and the participatory artists and researchers were introduced to the participants who were informed about the purposes of the study during recruitment.

\section{Sources of data}

This paper draws on data from the following sources:

- Qualitative semi-structured interviews, which were undertaken at baseline, at the end of the activity and three months after with a sub-set of participants 
and, in separate interviews, with their associated carers/family members. This was done in each of the four care homes in waves of three months. Included were sections on community belonging, relationships in the community and activities, including questions about the impact of the arts enrichment activity.

- Interview schedules, which included open-ended questions about connectivity and responses to the arts activity.

- Structured notes made by the participatory artists of their experiences of each of the arts enrichment activity sessions immediately after they were completed.

- Videos made of the arts enrichment sessions mainly used to enable conclusions to be checked.

- Socio-demographic and clinical data, e.g. age, occupational status, education, gender, medication use.

- The level of dementia, measured by the Clinical Dementia Rating (CDR) scale (Hughes et al. 1982), provides a context for qualitative judgements. For example, those with a higher score and therefore more advanced dementia might be expected to respond differently to the arts enrichment activity compared to those with a lower score. The CDR scale consists of scoring the categories of memory, orientation, judgement and problem solving, community affairs, home and hobbies, and personal care (CDR 0 healthy, CDR 0.5 questionable dementia, CDR 1 mild dementia, CDR 2 moderate dementia, CDR 3 severe dementia).

Newman and Goulding were present at the visual arts enrichment sessions and made structured field notes. The interviews ranged from a few minutes, for those who had become severely disabled by the condition and were unable to respond to questions, to 120 minutes duration. The interviews were digitally recorded and the recordings were professionally transcribed and anonymised for analysis. Participant names were replaced by alpha-numerical codes. These followed a fixed format: participants $=$ NDXX and carers $=$ NSYY, where ' $\mathrm{XX}$ ' and 'YY' are the numbers which distinguish individual respondents.

\section{Sample size}

In total 48 people with dementia and 37 carers/family members were recruited. Three carers/family members and three people in later life with dementia were interviewed in each of the four care homes in the three waves of interviews described above, which resulted in 72 transcripts. Two participatory artists were involved in all the activities in the four care homes and completed structured notes after each session.

\section{The visual arts enrichment activities}

The visual arts enrichment activities were undertaken for two hours a week for three months in four care homes. Activities were facilitated by two experienced participatory artists, reducing potential risks. The activity was either collective, with the group working towards a final product, such as a film or sculpture, or individual, such as a collage or painting, and as many senses as possible were engaged such 
as taste, touch and sound, especially with those with more severe dementia. A series of guidelines were produced to ensure that the activities had a level of commonality and were of high quality. The artists used an approach pioneered by Basting (2009), which emphasised creativity and looking forward, rather than focusing on memory losses or cognitive deficits. This aimed to create a positive enjoyable experience for those taking part. A visit to the Baltic Centre for Contemporary Art in Gateshead, UK, was organised for each of the four groups, and at the end of the three months of activities a celebratory event was held to which family members and carers were invited.

An example of an arts enrichment activity was one that was inspired by the work of Van Gogh (1853-1890). The room was dressed in a way that echoed the painting 'Starry Night' (1889) and the song 'Vincent (Starry, Starry Night)' (1971) by Don McLean was played in the background. This created a multi-sensory environment that provided the stimulation for the work that was created.

A further example is an activity based around imagination that was undertaken at one of the care homes and was described by one of the participatory artists in the following way:

I thought it would be good to include an active, imagination-based game of 'What will the imaginary ball become?' Everyone really engaged and got into it. It changed from a bird to a comb, to a Tarzan rope, to a boat ... ND32, an 87-year-old female who had a CDR score of 2 , moderate dementia, really enjoyed participating.

Despite the respondents' dementia, this exercise was successful and demonstrates that the capacity for imagination remained despite other losses. The participatory artists took a person-centred approach, organising the activity around the interests, abilities and energy levels of all those who took part. The participatory artists did not mention dementia and it did not feature in the creative responses. It is worth noting that those with severe dementia needed one-to-one support from carers and it was important to ensure that the artwork was created by the resident and not by the carer.

\section{Analysis}

The following categories (Morse 2008) were identified from the data:

- Creative expression.

- Increased communication.

- Self-esteem.

- Relationships with carers and family members.

The transcripts were coded using QSR NVivo with nodes which were emergent as coding progressed being identified through close multiple readings of the transcripts. An analysis of the resilience of people in later life with dementia living in care homes was not one of the original objectives of the Dementia and Imagination project. However, it became evident during the coding process that the data would support such an interrogation. It was not possible for all participants to comment on the findings, although some carers and family members attended a 
one-day seminar at Newcastle University, Newcastle upon Tyne, UK, for this purpose.

\section{Results}

The respondents in this study all had vulnerabilities, which included having dementia, of varying degrees and types, as well as other age-related issues such as macular degeneration and/or a lack of mobility. Therefore, they differed in the levels of adversity they experienced. As might be expected from such a diverse group, whether an individual was 'doing all right' (Braudy Harris 2008: 45) or not varied depending on the needs of that individual and whether those needs were being fulfilled. This situation was complicated because the symptoms of dementia might not only vary by the hour or day, but also worsen if the condition progressed over time. Some respondents became significantly worse over the six months of the data collection in each care home, while others appeared to have relatively stable symptoms. The characteristics of the sample are given in Table 1.

\section{Understanding resilience processes for older people with dementia through visual arts enrichment activities}

Creative expression within the arts enrichment activity

According to Simonton (2000), creativity, whether artistic or not, requires cognitive processes, personal characteristics, lifespan development and social contexts working together. For those with dementia, the first three might be expected to be compromised to some extent. However, it was apparent that the respondents were able to create art individually or collectively, and were able to access a range of resources, including past identities, to help them express a creative response to a particular topic, despite losses associated with their condition.

An example was a poem created by the participants in Care Home 3 and facilitated by the participatory artists on the theme of the sea. This care home was on the coast of North East England and residents could see the sea through their windows. They only had direct experience of the shore through infrequent trips organised by the home or family members. A poem, described by the participatory artists as 'a poetic, deep and dark meditation on the sea, ${ }^{8}$ was created from words produced by the residents. It was narrated by ND28, a female aged 87 years old with a CDR score of 1 , mild dementia, who had been a teacher. It was then overlain on film footage of the sea by the participatory artists. The video was five minutes long and the first 20 lines are given below.

\section{The Cruel Sea}

The beautiful sea goddess

Godiva Pearl

Beautiful ruffles

The ripples

Beautiful colouring

There's blue, black, white and a tinge of pink

Ooooo that's lovely 
Table 1. Baseline characteristics of the study sample

\begin{tabular}{|c|c|c|}
\hline & \multicolumn{2}{|c|}{ Care home sample } \\
\hline & $\mathrm{N}$ or mean & $\%$ or SD \\
\hline $\mathrm{N}$ & 48 & \\
\hline Mean age (SD) & 84.9 & 7.4 \\
\hline Female & 35 & 72.9 \\
\hline Male & 13 & 27.1 \\
\hline \multicolumn{3}{|l|}{ Marital status: } \\
\hline Married/co-habiting & 10 & 21.3 \\
\hline Single & 3 & 6.4 \\
\hline Widowed & 30 & 63.8 \\
\hline Divorced/separated & 4 & 8.5 \\
\hline \multicolumn{3}{|l|}{ Ethnicity: } \\
\hline White & 44 & 95.7 \\
\hline Asian/Asian British & - & - \\
\hline Other & 2 & 4.2 \\
\hline Mean age leaving full-time education (SD) & 15.8 & 2.7 \\
\hline \multicolumn{3}{|l|}{ Educational level: } \\
\hline Low & 23 & 47.9 \\
\hline Middle & 4 & 8.3 \\
\hline High & 8 & 16.7 \\
\hline \multicolumn{3}{|l|}{ Level of main activity/occupation (SES): } \\
\hline Unskilled & 3 & 6.3 \\
\hline Partly skilled & 3 & 6.3 \\
\hline Skilled (manual) & 16 & 33.3 \\
\hline Skilled (non-manual) & 9 & 18.8 \\
\hline \multicolumn{3}{|l|}{ Managerial/technical } \\
\hline Professional & 9 & 18.8 \\
\hline Mean level of main activity/occupation (SES 0-5) (SD) & 2.7 & 1.5 \\
\hline \multicolumn{3}{|l|}{ CDR scale: } \\
\hline 0.5 Questionable & 6 & 12.5 \\
\hline 1 Mild & 18 & 37.5 \\
\hline 2 Moderate & 8 & 16.7 \\
\hline 3 Severe & 16 & 33.3 \\
\hline \multicolumn{3}{|l|}{ Use of dementia- or mood-related medication: } \\
\hline None & 31 & 67.4 \\
\hline
\end{tabular}


Table 1. (Continued.)

\begin{tabular}{lcc}
\hline & \multicolumn{2}{c}{ Care home sample } \\
\cline { 2 - 3 } & $\mathrm{N}$ or mean & $\%$ or SD \\
\hline Only dementia-related & 11 & 23.9 \\
\hline Only mood-related & 4 & 8.7 \\
\hline Both & 0 & \\
\hline
\end{tabular}

Notes: Answers were not available for all questions, e.g. education level which even family members were not always able to provide, therefore totals are different in some sections. SD: standard deviation. SES: socio-economic group. CDR: Clinical Dementia Rating.

There are gorgeous colours there

Mostly blue and white, black and grey

Blue and more blue

The blue of the sky is different to the blue of the sea

When you are going past you say...

Ooooo lovely

The Sea is Sunday morning with two mothers and three children

It was cold but we didn't notice

Giant waves

Flashes of white

Makes me feel small

You wonder where they all come from

The waves come from the bottom of the deep blue sea.

In order to produce the poem respondents needed to be able to master a number of complex cognitive processes, bringing together available resources such as memory and an emotional response. They then had to configure them collectively, which involved a range of social skills, in a way that was new for this particular purpose. In this situation it is difficult to make a distinction between individual resources (human capital) and community or social resources (social capital) for resilience (Wild, Wiles and Allen 2013) within the group itself as both needed to be brought to bear and it was not possible to distinguish between them. While the distinction between individual and social resources for resilience is theoretically useful in some contexts, it is not visible when collective artwork is being produced.

Because members of the group producing the poem were of a similar age, between 82 and 91, they had broadly similar responses to the topic of the poem. For example, participants spoke of going swimming before the Second World War as children. These responses were influenced by their lifecourse experience and the social, psychological and physical effects of ageing. Having common memories and social attitudes provides a resource for social interactions. This is illustrated through the background verbal responses, heard in the video, to ND28's reading of the poem, affirming what is being said. This has the effect of producing a group and helps to create a collective resource that can be used in this context. ${ }^{9}$ This is analogous with the ways that a particular culture or tradition support 
resilience by providing perceived strengths to draw upon as explored in the context of Hawaii by Browne, Mokuau and Brawn (2009) and the Sami by Alex (2015).

The above narrative is only minimally about authenticating past identities in the present. For example, the line 'The Sea is Sunday morning with two mothers and three children. It was cold but we didn't notice' (Norrick 2009). Instead, it was more an expression of respondents' current emotional response to the topic. Creativity and self-expression is mentioned by Kitwood and Bredin (1992) as one of 12 indicators of relative wellbeing supporting personhood. They suggest that such an approach, described as 'rementing', might enable those with dementia to do better than might be expected given the progression of their dementia. Here, creative activities can be seen to make respondents more resilient while undertaking the activity than they might have been otherwise.

An essential part of creating the poem required emotional reflectivity (Boyle and Warren 2017). The ability to imagine what others were thinking and feeling so as to interpret their actions was achieved by all those involved, despite some having more advanced dementia. The existence of a social self 'rooted in family and community life' (Boyle and Warren 2017: 4) was demonstrated, as well as the ability to convey meanings through emotions.

\section{Increased communication within the visual arts enrichment activity}

An example of how the visual arts enrichment activity enabled communication between residents was ND34 who lived in Care Home 3, an 85-year-old female with an overall CDR score of 1 but with some component scores of 3 . Attempts to interview her were unsuccessful as she tended to reply with one-word answers repeating aspects of the question. She became less verbal over the period of the research project. She was normally dressed as if she was about to go out, wearing a coat and hat, and was unsettled, tending to wander about the care home not interacting much with other residents and care staff. NS26, a director of Care Home 3, states about her response, at T2, the end of the activity:

It really did feel quite different to me all of the activities were bringing everybody together, I had a greater interaction with ND34 that I'd ever had before. She was dancing with me and singing, and we were having a really great time, she was giving me cuddles and she'd give me a kiss. She was really connecting with other people as well in the group as well. Her whole body language seemed to be different.

For this person, normal one-to-one verbal communication had become difficult because of the progression of her dementia. However, within the visual arts activity, which involved painting life-size prints of each resident, she was able to express herself physically. The artists' notes state 'participant ND34, whilst painting was passionate and posing as she did so - her marks were dramatic and rhythmic'. The singing was instigated by the residents at the end of the session and was enjoyed by residents, family members and care staff.

Within the group she was observed from the video recording to be much more socially fluent, being able to interact with others, both with carers and other group members, than she did normally when observed in the care home. In this instance, the context of the activity supported her selfhood more than her normal situation 
and so supported resilience through facilitating non-verbal forms of communication. This individual was drawing upon both collective group knowledge of words to songs, and individual resources, for example, her remaining sense of self, for resilience, although it was difficult to distinguish between them empirically. However, it can be argued that collective resources were compensating for individual losses, or alternatively individual resources became more available within the social context of the art activity. Social relations also are important for the selfesteem of respondents (Boyle and Warren 2017), other aspects of which are explored below.

\section{Self-esteem within the arts enrichment activity and its relationship to resilience}

Coleman, Ivani-Chalian and Robinson linked self-esteem with resilience. They described a process of adaptation where:

Loss of self-esteem may result in greater rigidity, an inability to find coping strategies for a changing situation. Continuing high self-esteem may indicate that adaptation has occurred. (Coleman, Ivani-Chalian and Robinson 1993: 172)

The self-esteem of respondents and related ability to adapt could be either supported or undermined by the arts enrichment activity. An example of adaptability being supported was participant ND2, an 86-year-old female with a CDR score of 3, severe dementia, who lived in Care Home 1. She was observed on video to be smiling and to be pleased when she was praised by the participatory artists for her work. However, participant ND1, a 70-year-old male with a CDR score of 3, severe dementia, became frustrated in a session when he could not use a digital camera. However, overall NS8, an experienced manager of Care Home 1, stated at T2, that he 'was consistent in attending every week and I would say he benefited most out of all of the residents'. ND1 was demonstrating a loss of self-esteem in a particular context but also adaptability overall in relation to attending the arts enrichment activities.

Others became frustrated when they were unable to join in in the way they would like because of medical issues, often unrelated to dementia. An example was participant ND23, an 81-year-old male with a CDR score of 1 , mild dementia, who found it difficult to speak because of surgery for cancer of the throat which left him struggling to communicate. Attempting to engage in the arts enrichment activities had drawn attention to deficits that otherwise might have not been an issue. However, he overcame his communication problems through developing a strong relationship with the participatory artists and becoming less conscious of the fact that his speech was difficult to understand. He soon enjoyed the experience of the arts enrichment activities, demonstrating adaptation and self-esteem, and he liked the fact that this was part of a university research project. This situation shows how individual resources for resilience were supported by the social context of the arts enrichment activity. Successful adaptation might be viewed as being analogous to the mastery that is suggested by Cohen (2009) as being an important outcome of arts activities.

The wider impact of the arts enrichment activities upon carers and family members There were examples where the arts enrichment activities changed the carers and family members' perceptions of what participants were capable of and this, in 
turn, influenced their relationship with residents. In her text response to the T2 questions, NS26, a director of Care Home 3, stated:

I loved hearing people read, and was surprised how confident the readers were. I suppose I'd underestimated how capable people with dementia are and had assumed they would find this difficult. You underestimate people don't you, you think 'oh they're not going to do that'. The sessions allowed people to achieve their full potential as they were no assumptions that they may not be able to do things.

Despite having contact with the respondents on almost a daily basis, carers and family members underestimated what they were able to achieve. The participatory artists preferred family members not to be present during workshops as they felt that they could discourage participants from reaching their potential by doing tasks for them. NS14, an activity co-ordinator in Care Home 2, made the following comments about the relatives of ND19, a 75-year-old with a CDR of 1 (mild dementia) three months after the activity:

Without being awful, because I know they've just got her best interests at heart but I, I do think they thought that she wasn't capable of things she is capable of. And maybe by saying, 'Oh no ND19 wouldn't do something like that.' I think she's probably being able to enjoy it more without them there, you know.

As is noted by Kitwood (1993: 543), paraphrasing Sabat and Harre (1992), 'if a dementia sufferer is to sustain his or her part in the social world other people, with their corresponding expectations and performances are required'. Therefore, carers or family members underestimating the ability of residents to make decisions or failing to communicate effectively with them can undermine their personhood. In this situation, ND19's personhood is undermined by well-meaning family members whose approach reduced what she is able to achieve on her own.

There was also the potential for changing the relationship between carers and residents as NS26, a director of Care Home 3, points out in the T2 text responses:

The final celebration was great - we all enjoyed dancing and singing. It just felt like any social occasion/party - friends enjoying themselves, no distinction between those who were experiencing dementia and carers, family and friends.

NS26 was aware that while caring for residents a parent-child relationship can develop with those with dementia and this was something she wished to avoid. A more equal relationship between residents and carers was apparent at the celebration event, supporting the personhood and so resilience of participants.

\section{Discussion}

The analysis presents a picture of how resilience for people in later life with dementia may either be supported or undermined. This situation is more complex than is implied by Braudy Harris' (2008: 45) view that the person needs to have adversity to overcome and be 'doing all right'. Respondents might be 'doing all right' in 
particular contexts and less so in others and this situation was, for some, fluid. The arts enrichment activity mainly, although not exclusively, supported personhood and therefore respondents' resilience. Dividing resources for resilience into personal (human capital) and social or community (social capital) seems to underplay the complexity that was observed. The data suggest that as the art enrichment activity required both, in the collectively and individually produced artwork, it seems more appropriate to think of respondents' habitus in this context rather than their capital (Bourdieu 1984; Putnam 2000). The aspects of habitus involved include embodiment, where the 'body is in the social world and the social world is in the body' (Reay 2004: 432), where it 'predisposes individuals towards certain ways of behaving' (2004: 433) while allowing agency, being a compilation of collective and individual trajectories and being a complex interplay between past and present. In future work, it would be interesting to use this as the basis for an analysis of the Dementia and Imagination data-set as much of the habitus of the respondents with dementia remained intact.

As is stated in the theoretical framework, there is very little written about the process of adaptation or the 'ordinary magic' of resilience (Masten 2001). However, this process must involve creating a positive sense of identity that overrides negative factors and involves drawing from a range of resources, relying more on the collective aspects of habitus as a result of communication losses. Artistic creativity, which appeared normal and easy for all, may be seen as an adaptive, expressive response to how participants were experiencing their lives and both the conscious and unconscious challenges they faced. However, the data collection, art activity and analysis does not provide a narrative about what having dementia means as the condition was not mentioned, by respondents or participatory artists, and it did not appear in the art that was created by any of the respondents from the care homes. It is possible, although more research is needed, that individual and collective creative expression is the everyday process of adaptation, which is normally hidden, becoming visible. As Bond and Corner (2001: 103) state: 'living with dementia involves the active creation and re-creation of meaning and identity, and the negotiation of empowerment, as part of the daily work of living with the condition'. It is suggested that the process of normative adaption is the motivating force behind the creation and re-creation of meaning and identity.

The observed adaption process supporting resilience, described above, can be seen to be analogous with the normal identity change processes that healthy people in later life undergo, such as that described by Whitbourne, Sneed and Skultety (2002) as identity assimilation and identity accommodation. This is where change to personhood or identity might be resisted or accommodated in response to laterlife transitions, such as retirement or possibly the onset of dementia. These authors suggest that those who can maintain a balance between assimilation and accommodation are best placed to age well (Newman, Goulding and Whitehead 2012, 2013). Although, the resources available for these process to be undertaken might be reduced or changed, because of the effects of dementia, there is no reason to think that because a person has the condition that these identity processes are not relevant. However, they are difficult to see empirically.

The arts enrichment activities provide a context where respondents, even with severe dementia, collectively supported each other's personhood and so resilience. 
Another way of exploring this topic is illustrated in a review of the literature on the role of social ties in the resilience of those with chronic diseases by Janicki-Deverts and Cohen (2011). The authors state that aspects of social relationships regulate emotions, cognitions and behaviour 'preventing the extreme response associated with dysfunction' (Janicki-Deverts and Cohen 2011: 76), a mechanism that would be interesting to explore in the context of people with dementia with cognitive loss, communication difficulties and sometimes loss of inhibition. Those who were still able to read social situations well might benefit more than those who could not. A greater knowledge of the significance of social relationships would help social care practitioners in their efforts to improve the lives of those with dementia.

Within the arts enrichment activity carers and family members often underestimated what the residents were able to achieve and assumed they knew the residents' wishes. The resultant excess disability, a 'functional incapacity greater than that warranted by cognitive impairment', unfortunately may become permanent over time (Rogers et al. 2000: 268). It was evident that some residents were functioning at a higher level in the arts enrichment activity than they were at other times, so staff and family members became more aware of their potential capacities. Excess disability can be seen as inhibiting adaptation and so making participants less resilient than they might have been.

\section{Conclusion}

From this study it is possible to conclude that those with severe dementia are capable of demonstrating resilience and that the arts enrichment activity facilitates this through supporting personhood in the domains of creative expression, communication, self-esteem, and through its effects on carers and family members. It is evident that dividing up resources for resilience into individual and social forms is unhelpful when analysing respondents' responses to the arts enrichment activity. A more complex picture is produced where resources appear to be more related to the respondent's habitus which is operationalised in a specific way within the arts enrichment activity. It is possible that the creative response observed in the visual arts enrichment activity is the normal adaptive process (Masten 2001) being undertaken in response to the threats to resilience being experienced. The difference between this group and healthy older people is that the threats to resilience and the resources that may be brought to bear are very different.

\section{Notes}

1 See https://www.alzheimers.org.uk/info/20025/policy_and_influencing/251/dementia_uk (accessed 16 November 2017).

2 See http://dementiaandimagination.org.uk/ (accessed 16 November 2017).

3 See http://www.ahrc.ac.uk/research/fundedthemesandprogrammes/crosscouncilprogrammes/connectedcommunities/ (accessed 16 November 2017).

4 'A standing or status that is bestowed upon one human being by others' (Kitwood 1997: 8).

5 An issue that is common in sociological studies of people in later life (see e.g. Newman, Goulding and Whitehead 2013). 
6 Interestingly, there appears to be more work on the resilience of care-givers for those with dementia than work on older people with dementia themselves, such as Harmell, Chattillion and Roepke (2011) and Donnellan, Bennett and Soulsby (2015).

7 See http://www.newdynamics.group.shef.ac.uk/ (accessed 16 November 2017).

8 See https://vimeo.com/124503277 (accessed 16 November 2017).

9 The idea that the creation of art is fundamentally social is suggested by Bourdieu (1993), who criticises what he describes as a charismatic ideology of art production meaning that art originates from the individual creator with no reference to how the creator was created.

Acknowledgements. The authors would like to thank the participants in the project. Regional and national collaborating partners in Dementia and Imagination are the following: Age Watch, Alzheimer's Society, the Arts Council of Wales, Denbighshire County Council, Engage Cymru, Derbyshire Community Health Service NHS, Equal Arts, the Baltic Centre for Contemporary Art, Tyne and Wear Archives and Museums, and Nottingham Contemporary Ltd.

Funding. Dementia and Imagination was funded as 'Dementia and imagination: connecting communities and developing well-being through socially engaged visual arts practice' (grant reference AH/K00333X/1), by the Economic and Social Research Council and the Arts and Humanities Research Council as a part of the Cross-Council Connected Communities Programme.

Ethical approval. The study received UK National Health Service ethical approval from the North Wales Research Ethics Committee - West (reference number 13/WA/0365).

\section{References}

Alex L (2015) Resilience among old Sami women. Ageing \& Society 36, 1738-1756.

Basting A (2009) Forget Memory: Creating Better Lives for People with Dementia. Baltimore, Maryland: Johns Hopkins University Press.

Basting A and Killick J (2003) The Arts and Dementia Care: A Resource Guide. New York.: Center for Creative Aging.

Bennett K, Reyes-Rodriguez M, Altamar P and Soulsby L (2016) Resilience amongst older Colombians living in poverty: an ecological approach. Journal of Cross-cultural Gerontology 31, 385-407.

Bond J and Corner L (2001) Researching dementia: are there unique methodological challenges for health services research? Ageing \& Society 21, 95-116.

Bourdieu P (1993) Outline of a sociological theory of art perception. In Bourdieu P (ed.), The Field of Cultural Production. Cambridge: Polity Press, pp. 215-237.

Bourdieu P (1984) Distinction: A Social Critique of the Judgement of Taste. Cambridge, Massachusetts: Harvard University Press.

Boyle G and Warren L (2017) Showing how they feel: the emotional reflexivity of people with dementia. Families, Relationships and Societies 6, 3-19.

Braudy Harris B (2008) Another wrinkle in the debate about successful aging: the undervalued concept of resilience and the lived experience of dementia. International Journal of Ageing and Human Development 67, 43-61.

Bronfenbrenner U (1994) Ecological models of human development. In Husen T and Postlethwaite T (eds), International Encyclopaedia of Education. Volume 3, second edition, New York: Elsevier.

Browne C, Mokuau N and Brawn K (2009) Adversity and resilience in the lives of native Hawaiian elders. Social Work 54, 253-261.

Buettner L and Fitzsimmons S (2003) Activity calendars for older adults with dementia: what you see is not what you get. American Journal of Alzheimer's Disease and Other Dementias 18, 215-226.

Claire L, Kinsella G, Logsdon R, Whitlatch C and Zarit S (2011) Building resilience in mild cognitive impairment and early-stage dementia: innovative approaches to intervention and outcome evaluation. In Resnick B, Gwyther L and Roberto K (eds), Resilience in Aging: Concepts, Research, and Outcomes. New York: Springer, pp. 245-259. 
Claire L, Rowlands J, Bruce E, Surr C and Downs M (2008) The experience of living with dementia in residential care: an interpretative phenomenological analysis. The Gerontologist 48, 711-720.

Clarke C and Bailey C (2016) Narrative citizenship, resilience and inclusion with dementia: on the inside or on the outside of physical and social places. Dementia 15, 434-452.

Cohen G (2006) Research on creativity and aging: the positive impact of the arts on health and illness. Generations 1, 7-15.

Cohen G (2009) New theories and research findings on the positive influence of music and art on health with aging. Arts and Health 1, 48-62.

Coleman P, Ivani-Chalian C and Robinson M (1993) Self-esteem and its sources: stability and change in later life. Ageing \& Society 13, 171-192.

Donnellan W, Bennett K and Soulsby L (2015) What are the factors that facilitate or hinder resilience in older spousal dementia carers? A qualitative study. Aging and Mental Health 19, 932-939.

Downs M (2000) Dementia in a socio-cultural context: an idea whose time has come. Ageing \& Society 20, 369-375.

Fisher B and Specht D (1999) Successful aging and creativity in later life. Journal of Aging Studies 13, 457472.

Harmell A, Chattillion E and Roepke S (2011) A review of the psychobiology of dementia caregiving: a focus on resilience factors. Current Psychiatry Reports 13, 219-224.

Hope S (2016) Bursting paradigms: a colour wheel of practice-research. Cultural Trends 25, 74-86.

Hughes C, Berg L, Danziger W, Coben L and Martin R (1982) A new clinical scale for the staging of dementia. British Journal of Psychiatry 140, 566-572.

Janicki-Deverts D and Cohen S (2011) Social ties and resilience in chronic disease. In Southwick S, Litz B, Charney D and Friedman M (eds), Resilience and Mental Health: Challenges Across the Lifespan. Cambridge: Cambridge University Press, pp. 76-89.

Kitwood T (1993) Person and process in dementia. International Journal of Geriatric Psychiatry 8, 541545.

Kitwood T (1997) Dementia Reconsidered. Buckingham, UK: Open University Press.

Kitwood T and Bredin K (1992) Towards a theory of dementia care: personhood and well-being. Ageing \& Society 12, 269-287.

Mariske M and Willis S (1998) Practical creativity in older adults' everyday problem solving: life span perspectives. In Adams-Price CE (ed.), Creativity and Successful Aging: Theoretical and Empirical Approaches. New York: Springer, pp. 73-113.

Masten A (2001) Ordinary magic: resilience processes in development. American Psychologist 56, 227-238.

McFadden S and Basting A (2010) Healthy aging persons and their brains: promoting resilience through creative engagement. Clinics in Geriatric Medicine 26, 149-161.

Mental Capacity Act (2005) Mental Capacity Act 2005. London: HMSO. Available at http://www.legislation.gov.uk/ukpga/2005/9/pdfs/ukpga_20050009_en.pdf (Accessed 15 November 2017).

Morse J (2008) Confusing categories and themes. Qualitative Health Research 18, 727-723.

Murray M, Amigoni D, Bernard M, Crummett A, Goulding A, Munro L, Newman A, Rezzano J, Rickett M, Tew P and Warren $\mathbf{L}$ (2014) Understanding and transforming ageing through the arts. In Walker A (ed.), New Science of Ageing. Poole, UK: Policy Press, pp. 77-112.

Newman A, Baber M, O'Brien D, Goulding A, Hedd Jones C, Howson T, Jones C, Parkinson C, Taylor K, Tischler V and Windle G (2016) Carrying out research across the arts and humanities and social sciences: developing the methodology for Dementia and Imagination. Cultural Trends 25, 218-232.

Newman A, Goulding A and Whitehead C (2012) The consumption of contemporary visual art: identity formation in late adulthood. Cultural Trends 21, 29-45.

Newman A, Goulding A and Whitehead C (2013) How cultural capital, habitus and class influence the responses of older adults to the field of contemporary visual art. Poetics 41, 456-480.

Norrick N (2009) The construction of multiple identities in elderly narrators' stories. Ageing \& Society 29, 903-927.

Putnam R (2000) Bowling Alone: The Collapse and Revival of American Community. New York: Simon and Schuster.

Reay D (2004) 'It's all becoming a habitus': beyond the habitual use of habitus in educational research. British Journal of Sociology of Education 25, 431-444. 
Rogers J, Holm M, Burgio L, Hsu C, Hardin M and McDowell B (2000) Excess disability during morning care in nursing home residents with dementia. International Psychogeriatrics 12, 267-282.

Roush R, Braun M, Basting A, Winakur J, Rosenberg F and McFadden S (2011) Using the arts to promote resiliency among persons with dementia and their caregivers. In Resnick B, Gwyther L and Roberto K (eds), Resilience in Aging: Concepts, Research, and Outcomes. New York: Springer, pp. $105-120$.

Rowe J and Kahn R (1987) Human aging: usual and successful. Science 237, 143-149.

Sabat S and Harre R (1992) The construction and deconstruction of self in Alzheimer's disease. Ageing \& Society 12, 443-461.

Simonton D (2000) Creativity: cognitive, personal, developmental, and social aspects. American Psychologist 55, 151-158.

Whitbourne S, Sneed J and Skultety K (2002) Identity processes in adulthood: theoretical and methodological challenges. Identity 2, 29-45.

Wild K, Wiles J and Allen R (2013) Resilience: thoughts on the value of the concept for critical gerontology. Ageing \& Society 33, 137-158.

Wiles R (2013) What Are Qualitative Research Ethics? London: Bloomsbury.

Windle G (2011) What is resilience? A review and concept analysis. Reviews in Clinical Gerontology 21, $152-169$.

Windle G (2012) The contribution of resilience to healthy ageing. Perspectives in Public Health 132, $159-160$.

Windle G and Bennett K (2011) Resilience and caring relationships. In Ungar M (ed.), The Social Ecology of Resilience. New York: Springer, pp. 219-232.

Windle G, Newman A, Burholt V, Woods B, O'Brien D, Baber M, Hounsome B, Parkinson C and Tischler V (2016) Dementia and Imagination: a mixed-methods protocol for arts and science research. BMJ Open 6, e011634.

Young R, Tischler V, Hulbert S and Camic P (2015) The impact of viewing and making art on verbal fluency and memory in people with dementia in an art gallery setting. Psychology of Aesthetics, Creativity, and the Arts 9, 368-375.

Cite this article: Newman A, Goulding A, Davenport B, Windle G (2019). The role of the visual arts in the resilience of people living with dementia in care homes. Ageing \& Society 39, 2465-2482. https://doi.org/ $10.1017 /$ S0144686X18000594 